\title{
Heart failure: Pilot transcriptomic analysis of cardiac tissue by RNA-sequencing
}

\author{
Concetta Schiano ${ }^{1}$, Valerio Costa ${ }^{2}$, Marianna Aprile ${ }^{2}$, Vincenzo Grimaldi ${ }^{3}$, \\ Ciro Maiello ${ }^{4}$, Roberta Esposito ${ }^{2}$, Andrea Soricelli ${ }^{1,5}$, Vittorio Colantuoni ${ }^{6}$, \\ Francesco Donatelli ${ }^{7}$, Alfredo Ciccodicola ${ }^{2,8}$, Claudio Napoli ${ }^{1,3}$ \\ ${ }^{1}$ IRCCS SDN, Naples, Italy
}

${ }^{2}$ Institute of Genetics and Biophysics "Adriano Buzzati-Traverso", National Research Council, Naples, Italy

${ }^{3}$ University of Studies of Campania "Luigi Vanvitelli”, Naples, Italy

${ }^{4}$ Department of Cardiothoracic Science, U.O.S.D. of Heart Transplantation, Monaldi Hospital, Naples, Italy

${ }^{5}$ Department of Motor Sciences and Healthiness, University of Naples "Parthenope", Naples, Italy

${ }^{6}$ Department of Science and Technology, University of Sannio, Benevento, Italy

${ }^{7}$ Department for Cardiovascular and Metabolic Disease, Division of Cardiothoracic Surgery,

Faculty of Medicine, University of Milan, Italy

${ }^{8}$ Department of Science and Technology, University of Naples "Parthenope", Naples, Italy

\begin{abstract}
Background: Despite left ventricular (LV) dysfunction contributing to mortality in chronic heart failure (HF), the molecular mechanisms of LV failure continues to remain poorly understood and myocardial biomarkers have yet to be identified. The aim of this pilot study was to investigate specific transcriptome changes occurring in cardiac tissues of patients with HF compared to healthy condition patients to improve diagnosis and possible treatment of affected subjects.

Methods: Unlike other studies, only dilated cardiomyopathy $(D C M)(n=2)$ and restrictive cardiomyopathy $(R C M)(n=2)$ patients who did not report family history of the disease were selected with the aim of obtaining a homogeneous population for the study. The transcriptome of all patients were studied by RNA-sequencing (RNA-Seq) and the read counts were adequately filtered and normalized using a recently developed user-friendly tool for RNA-Seq data analysis, based on a new graphical user interface (RNA-SeqGUI).

Results: By using this approach in a pairwise comparison with healthy donors, we were able to identify DCM- and RCM-specific expression signatures for protein-coding genes as well as for long noncoding RNAs (lncRNAs). Differential expression of 5 genes encoding different members of the mediator complex was disclosed in this analysis. Interestingly, a significant alteration was found for genes which had

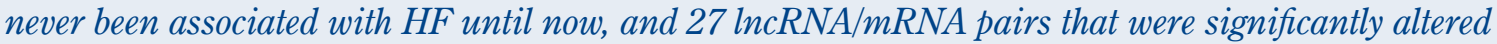
in HF patients.

Conclusions: The present findings revealed specific expression pattern of both protein-coding and lncRNAs in HF patients, confirming that new LV myocardial biomarkers could be reliably identified using Next-Generation Sequencing-based approaches. (Cardiol J 2017; 24, 5: 539-553)
\end{abstract}

Key words: cardiovascular disease, heart failure, noncodingRNA, mediator complex, RNA-sequencing

Address for correspondence: Dr. Concetta Schiano, MChem, PhD, IRCCS SDN, Via E. Gianturco 113, 80143, Naples, Italy, tel: (+39) 081 2408260, fax: (+39) 081 668841, e-mail: cschiano@sdn-napoli.it

Received: 27.09.2016 Accepted: 13.04.2017 


\section{Introduction}

Cardiomyopathies are a heterogeneous group of myocardial diseases resulting in cardiac dysfunction, which are clinically manifested with heart failure (HF). With the rapid evolution of molecular genetics in cardiology, the knowledge and literature of the complex interplay between genetics and cardiomyopathies have significantly expanded over the past few decades. Inherited cardiomyopathies include a wide spectrum of clinical phenotypes, which classically include dilated cardiomyopathy (DCM), hypertrophic cardiomyopathy (HCM), restrictive cardiomyopathy (RCM), and others [1]. DCM is characterized by an increase in both left ventricular (LV) mass and volume with thinning and stretching of LV wall [1]. HCM is characterized by inappropriate myocardial hypertrophy (without identifiable etiology such as hypertension, aortic stenosis or other contributing factors), interstitial fibrosis, myofiber disarray, disorganized myocardial architecture and impaired LV performance [1]. $\mathrm{RCM}$ is a heart-muscle disease resulting in impaired ventricular filling with normal or decreased diastolic volume of either or both ventricles [1] However, one phenotypic pattern may progress to another, or many others may manifest as more than one phenotype [2]. HF is a multi-factorial condition occurring in about $2-3 \%$ of the adult population [2-4]. The main cause is coronary artery disease (CAD) and it is a consequence of myocardial infarction (MI) [2-4]. The onset and progression of $\mathrm{HF}$ are closely related to several molecular and cellular alterations.

In recent years, advances in drug treatment have significantly improved the prognosis of $\mathrm{HF}$ patients. However, there are still several limitations on the benefits of medical therapy for patients with refractory end-stage disease, which often require mechanical support or heart transplantation. Thus, the development of novel and effective therapeutic treatments for $\mathrm{HF}$ is a major challenge today and requires a detailed knowledge of $\mathrm{HF}$ molecular pathogenesis.

Global gene expression profiling comparing disease vs. a healthy condition is a valuable approach for discovering new potential biomarkers for diagnosis/prediction of disease severity and for identifying novel drug targets. Particularly, whole-transcriptome analysis is increasingly acquiring a key role in the knowledge of mechanisms responsible for complex diseases, elucidating the involvement of multiple genes and pathways in pathological mechanisms.
Over the last decade, global gene expression analysis using microarrays has been widely applied to cardiovascular research [5, 6]. Most transcriptome studies on MI have been performed in rodents due to higher accessibility to homogenous populations and myocardial tissue at defined stages of the disease $[5,6]$. Although gene expression studies have elucidated many crucial molecular alterations involved in HF pathophysiology, they do not fully capture the complexity of human transcriptome [7]. Human myocardial tissue is difficult to obtain and usually comes from heterogeneous patient cohorts, generally in late-stage cardiomyopathies of different etiologies leading to HF. Despite these limitations, global gene expression analysis using microarrays has been widely applied to the field of cardiovascular research. However, although gene expression studies have elucidated many crucial molecular alterations involved in HF pathophysiology, they could not fully capture the complexity of human transcriptome [7]. The introduction of RNA-Sequencing (RNA-Seq) has overcome some drawbacks of previously used technologies, allowing a simultaneous investigation of different layers of transcriptome complexity at an in-depth level of resolution [8, 9]. Indeed, RNA-Seq analysis revealed alterations of cytoskeletal and nucleocytoplasmic transport-related genes, as well as of other key pathways in HF [7-12]. Interestingly, several studies have also highlighted the importance of noncoding RNAs (ncRNAs) in failing human hearts $[13,14]$. Currently, the best-characterized ncRNAs in the heart are the microRNAs (miRNAs), which finely modify mRNA expression through posttranscriptional silencing. Recently, several miRNAs have been found to be associated with cardiovascular disease (CVD) [13]. Functional analyses have demonstrated that several dysregulated miRNAs may exert either positive or negative regulatory effects on cardiac hypertrophic pathways. Indeed, miR-1, miR-133, miR-378, miR-185 and miR-155 showed anti-hypertrophic effects [15-18]; while miR-208 family, miR-212/132, miR-23 and miR-199 promoted hypertrophy in cardiomyocytes $[19,20]$. Unlike miRNAs, long noncoding RNAs (lncRNAs) are much less well characterized, and how they function in biology and gene regulation remains an attractive area of investigation [14].

In the last several years, some lncRNAs have been identified and their function has been implicated in different biological processes. However, only a few lncRNAs were associated to cardiomyopathy and heart biology, as myosin heavy chainassociated RNA transcript (MHRT) [21]. It is 
Table 1. Demographic and clinical characteristics of patients affected by cardiomyopathy.

\begin{tabular}{lcc}
\hline Characteristics & Donors data & Recipients data \\
\hline Subjects & 4 & 4 \\
Gender female & $3 / 4(77 \%)$ & $2 / 2(50 \%)$ \\
Age [years] & $57.5 \pm 11.7$ & $57.5 \pm 10.2$ \\
Waiting time on transplantation list [months] & Accidental death & $10 \pm 10.3$ \\
Body mass index $\left[\mathrm{kg} / \mathrm{m}^{2}\right]$ & $26.4 \pm 4.2$ & $27.1 \pm 3.3$ \\
Serum creatinine $[\mathrm{mg} / \mathrm{dL}]$ & $1.32 \pm 0.2$ & $1.4 \pm 0.1$ \\
Total cholesterol $[\mathrm{mg} / \mathrm{dL}]$ & $152.0 \pm 30.4$ & $177.0 \pm 43.0$ \\
Low density lipoprotein $[\mathrm{mg} / \mathrm{dL}]$ & $96.50 \pm 32.2$ & $108.2 \pm 27.7$ \\
Diabetes & No & No \\
Statin treatment & No & No \\
Smoking & No & No \\
\hline
\end{tabular}

Data are mean values \pm standard deviation

a cluster of lncRNAs transcribed from the MYH7 gene that encodes the $\beta \mathrm{MHC}$ protein. MHRT expression is heart specific and it is expressed at low levels in the fetal heart, increasing in the adult heart. Furthermore, higher levels of long intergenic RNA (lincRNA) predicting cardiac remodeling (LIPCAR) in plasma from HF patients following ischemic cardiomyopathy were independently associated with an elevated risk for future cardiovascular death and predictive for LV remodeling [22]. This effect was also reported for ANRIL, KCNQ1OT1, MIAT, and MALAT1 in a cohort of $414 \mathrm{MI}$ patients [23]. Nevertheless, the specific functions of these transcripts within the heart or vascular tissue remain relatively unknown. Moreover, ANRIL was shown to be highly expressed in atherosclerotic plaques and might be an accurate regulator in the inflammatory nuclear factor kappa B pathway [24]. Therefore, the importance of the altered expression of lncRNAs in human HF induces exploration of their putative involvement and functional roles in myocardial disease [24].

In this pilot study, a systematic transcriptome analysis was performed, by RNA-Seq on myocardial tissue specimens from HF patients vs. healthy donors (HD) in order to reveal evidence of a distinctive expression network signature in endstage HF diseased hearts. This approach may offer important insights into the complex pathogenesis of advanced cardiac failure, as well as for identifying potential targets for therapeutic intervention. Initially highlighted were classic heart diseaseassociated coding and noncoding genes, whose expression was significantly altered as MYH6, MYH7 and MHRT. Then, differentially expressed genes (DEGs) were screened and their possible roles in the pathogenesis of HF were explored by using multiple bioinformatics methods to identify specific transcriptomic signatures.

\section{Methods}

\section{Patients and tissue samples collection}

This study was performed according to the principles outlined in the Helsinki Declaration, and was approved by the local Ethics Committee. Eight heart biopsies were collected from heart transplantation candidates. The diagnosis of HF (DCM, $\mathrm{n}=2 ; \mathrm{RCM}, \mathrm{n}=2$ ) was determined by medical history, pathological and instrumental examination. LV tissue specimens ( $n=4$ for organ donors and $\mathrm{n}=4$ for recipients) were acquired during surgical intervention at the "Monaldi" Hospital. Cardiac tissue samples were harvested and snap frozen in liquid nitrogen at the time of cardiac surgery. Clinical features of patients are shown in Table 1.

\section{RNA extraction, library preparation and sequencing}

Heart tissue samples were homogenized by Tissue Lyser Disruption system (Qiagen) and total RNA was isolated with TRIzol Reagent (Invitrogen) according to manufacturer protocol. cDNA library preparation was performed starting from $4 \mu \mathrm{g}$ of total RNA using Illumina TruSeq Libraries and then sequenced at high coverage on the Illumina HiSeq2000 Next-Generation Sequencing (NGS) platform available at Tigem Institute in Pozzuoli (Naples) [25]. (RNA integrity and quality are detailed in Supplementary Methods - see journal website). 


\section{RNA-Seq data analysis}

The quality control on raw reads was performed using FastQC (http://www.bioinformatics. babraham.ac.uk/projects/fastqc/). High-quality reads were mapped to the human reference transcriptome (Ensembl v70) and to human reference genome (GRCh37/hg19) using TopHat2 v2.0.10 [26]. Only unique mapped reads were used to quantify gene expression in each sample. Gene expression as reads counts were estimated after filtering (Proportion test implemented in NOISeqBio package in $\mathrm{R}$ ) and normalization of raw reads counts using RNA-SeqGUI developed in R language [27]. Principal component analysis (PCA), mean average and density plots were generated using the graphical user interface (GUI). Differential expression between patients was evaluated using the non-parametric NOISeqBIO function [28] implemented in RNA-SeqGUI. A posterior probability $(\mathrm{PP})>0.95$ was used to determine DEGs. RNA-Seq datasets were submitted to GEO public resource and the accession number to the data files is GSE71613 (http://www.ncbi.nlm.nih.gov/ geo/query/acc.cgi? acc $=$ GSE71613).

\section{Gene ontology and pathway analysis}

The Kyoto Encyclopedia of Genes and Genomes (KEGG) (http://www.genome.ad.jp/kegg/) [29] is an authoritative database containing a variety of biochemical pathways. In addition, the Database for Annotation, Visualization and Integration Discovery (DAVID) (https://david.ncifcrf.gov) [30] is a gene functional classification tool that organizes and condenses abundant heterogeneous annotation content. Functional enrichment analysis was conducted in order to recognize the DEG enriched biochemical pathways using KEGG database, gene ontology (GO) associated biological functions and PANTHER Gene analysis tools version 10.0 (http:// www.pantherdb.org/tools) [31]. Furthermore, DAVID online tools were applied for the GO and KEGG pathway enrichment analyses with a p value set to $<0.05$ (according to Benjamini-Hochberg correction) were considered significantly enriched.

Because of a lack of strand specificity in the sequencing protocol, for the analysis of lncRNAs, only intergenic RNAs and antisense RNAs were selected - annotated in Ensembl v70 - excluding transcripts showing overlap with protein coding genes. Transcriptional start sites (TSSs) of lncRNAs and protein-coding genes were downloaded from Ensembl v70 annotation, using the tool Table Browser of UCSC Genome Browser (http://genome.ucsc.edu/cgi-bin/hgTables) [32].
Differentially expressed lncRNAs, between LV tissues from HF vs. HD samples, were associated with the nearest TSS of protein coding genes using the function "closestBed" of BEDTools [33] with default parameters.

\section{Data validation by qRT-PCR \\ and statistical analysis}

One microgram of total RNA, from HF patients and $\mathrm{HD}$, was reverse-transcribed to cDNA using the SuperScript ${ }^{\circledR}$ IV (Invitrogen), following the manufacturer's protocol. Primer sequences and corresponding polymerase chain reaction (PCR) conditions are shown in the Table S1 (see journal website, supplementary file). Gene expression was quantitatively determined by quantitative real-time PCR (qRT-PCR) analysis (detailed in Supplementary Methods - see journal website). Results were analyzed by threshold cycle (Ct) approach and normalized to RPS18, used as housekeeping gene [34]. Relative expression levels of the target genes were shown as fold change $(\mathrm{FC})$, calculated through the $2^{-\Delta \Delta \mathrm{Ct}}$ method [34]. Results are reported as mean \pm standard error of three single experiments. Differences between experimental groups were analyzed by the ANOVA and t Student's tests, considering significant a $\mathrm{p}$ value $<0.05(*)$ and particularly significant a $\mathrm{p}<0.01(* *)$.

\section{Results}

\section{Transcriptome analysis of cardiac tissues}

This pilot study investigates the changes occurring in the transcriptome of cardiac tissues in HF patients and healthy subjects by RNA-Seq technology. Clinical characteristics of individuals are reported in Table 1 . We isolated total RNA from LV myocardium of patients with DCM and $\mathrm{RCM}$ obtained at the time of heart transplantation. Control samples were obtained from transplant donor hearts. The cause of death in these individuals was road accident. All HD had no history of myocardial disease or active infections or significant comorbidities, such as hypertension, diabetes and hypercholesterolemia at the time of transplantation. Three of four subjects were female $(77 \%)$ with a mean age of $58 \pm 11.7$ years. Donors and recipients were of Caucasian ethnicity.

Globally, RNA-Seq produced paired-end reads with a sufficient quality and read coverage per sample to perform reliable gene expression analysis (Fig. S1A-C - see journal website) [35]. The expression levels of protein-coding and noncoding genes have been analyzed for all samples. 


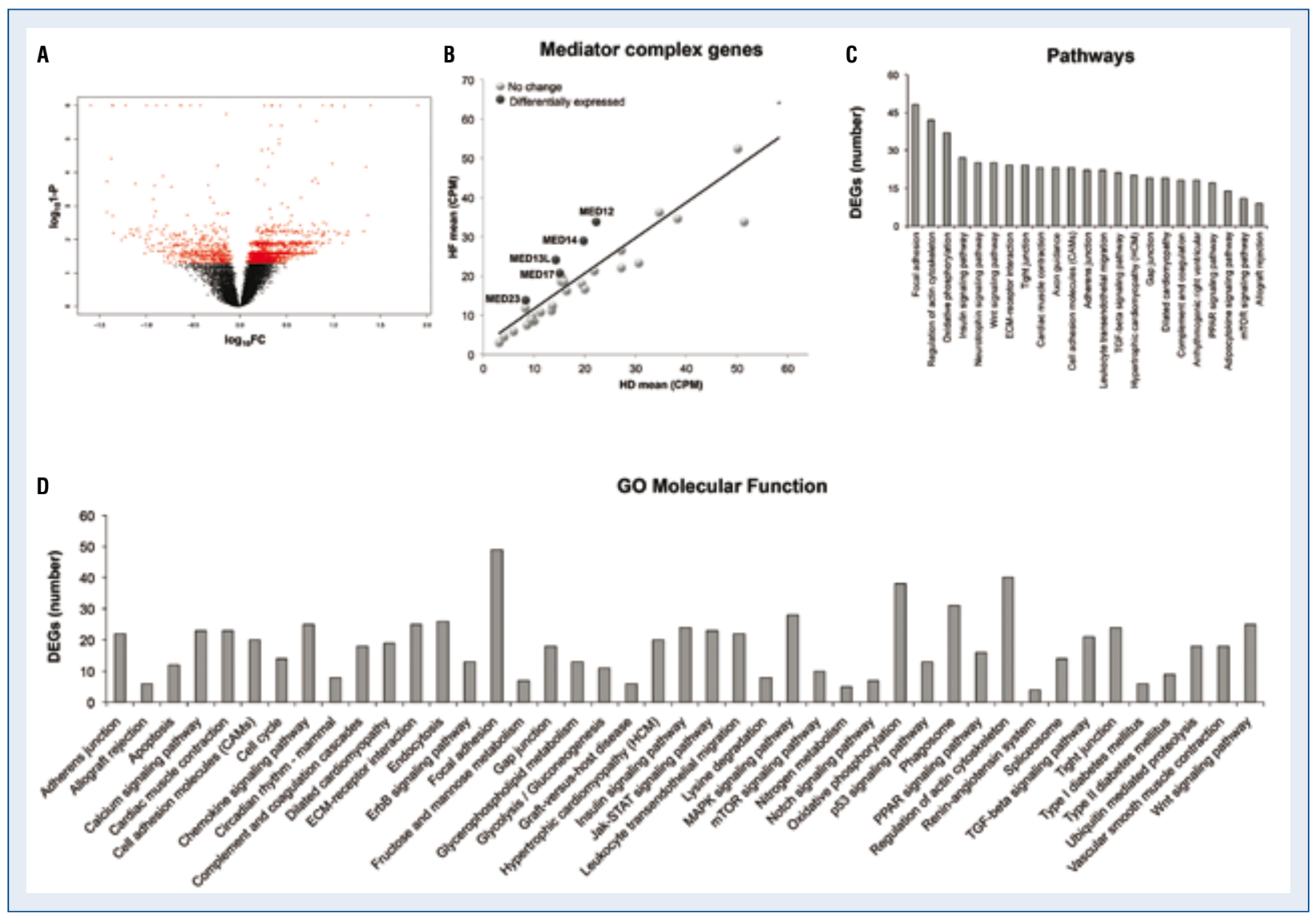

Figure 1. Analysis of differentially expressed genes in heart failure (HF) and heart donor (HD) groups. A. Volcano plot reporting on the $y$ axis 1-P (posterior probability) in log10 scale and on the $x$ axis $\log _{10} F C$ (fold change calculated as disease/healthy samples). Genes identified as significantly differentially expressed (PP $>0.95$ ) are shown as red dots; B. Scatter plot of normalized (counts per million [CPM]) mediator complex (MED) gene expression values. Each circle represents a unique gene encoding a mediator complex family member. Black circles indicate differentially expressed gene (DEGs); C, D. Bar graph reporting the results of the "Pathway" and "Gene Ontology (GO) Molecular Function" analysis used to infer the function of DEGs identified by RNA sequencing. Categories are reported on the $x$ axis, DEGs' number on the $y$ axis.

Multidimensional scaling analysis confirmed high correlation and reproducibility among individual samples of each group (Fig. S1D - see journal website). PCA revealed that the two different groups of patients, with different HF etiology (DMC/RCM), significantly differed (Fig. S1E see journal website). RNA-Seq revealed that about 15,800 genes (Ensembl v70) were expressed in heart specimens and about 500 of them are currently annotated as lncRNAs by the GENCODE Consortium. As the classification of lncRNAs is far from complete and most of these transcripts overlap protein-coding loci, the focus was specifically on the lincRNAs and antisense lncRNAs that can be unequivocally quantified by RNA-Seq. As a general observation there were significant differences between HF and HD transcriptomes, an attempt was made to identify genes with differential expression that were potentially associated with disease etiology.

Significant alterations in the protein-coding transcriptome of $\mathrm{HF}$ vs. HD

RNA-SeqGUI allows virtually quantifying all expressed genes in a cell or tissue [15]. The initial determination for each sample was the expression level of 56,622 loci (Ensembl v70), then the focus turned to the identification of DEGs between the groups under evaluation. First, we compared the gene expression profiles of HF vs. HD; next, DCM vs. HD; finally, RCM vs. HD subjects.

Data analysis revealed that 2,428; 470 and 3,685 genes were significantly altered in the three groups, respectively (Fig. 1A). The degree of differential expression was variable and ranged from +6.3 to $-5.3 \log 2 \mathrm{FC}$ between $\mathrm{HF}$ and $\mathrm{HD}$; 
Table 2. Top 10 up-regulated and down-regulated genes

\begin{tabular}{|c|c|c|c|c|c|}
\hline \multicolumn{2}{|c|}{ HF top 10 DEGs } & \multicolumn{2}{|c|}{ DCM top 10 DEGs } & \multicolumn{2}{|c|}{ RCM top 10 DEGs } \\
\hline Up-regulated & $\log 2 F C$ & Up-regulated DEGs & $\log 2 F C$ & Up-regulated DEGs & $\log 2 \mathrm{FC}$ \\
\hline HAPLN1 & 6.31 & MTRNR2L1 & 10.56 & HAPLN1 & 6.41 \\
\hline CFAP61 & 4.64 & HAPLN1 & 6.21 & CFAP61 & 5.05 \\
\hline COL9A1 & 4.54 & MYOZ1 & 5.32 & COL9A1 & 5.00 \\
\hline MYOZ1 & 4.47 & FOSB & 4.49 & CENPA & 4.08 \\
\hline HAND1 & 4.39 & HAND1 & 4.20 & KIAA1211 & 4.07 \\
\hline KIAA1211 & 3.84 & CFAP61 & 4.05 & ASB18 & 3.96 \\
\hline UNC80 & 3.83 & SYT12 & 3.92 & UNC80 & 3.86 \\
\hline TNN & 3.76 & COL9A1 & 3.85 & RHCG & 3.80 \\
\hline COMP & 3.71 & UNC80 & 3.81 & COMP & 3.69 \\
\hline CENPA & 3.65 & COMP & 3.73 & WBSCR17 & 3.55 \\
\hline Down-regulated & $\log 2 F C$ & Down-regulated & $\log 2 F C$ & Down-regulated & $\log 2 F C$ \\
\hline TRAJ39 & -5.30 & TRAJ39 & -5.55 & RSPO4 & -5.92 \\
\hline TRAJ49 & -4.72 & CTRB1 & -4.94 & EDN2 & -5.74 \\
\hline CTRB1 & -4.72 & CXCL14 & -4.59 & SELE & -5.70 \\
\hline PGA4 & -4.57 & TRAJ49 & -4.36 & MYOG & -5.40 \\
\hline ADAMTS8 & -4.54 & PGA5 & -4.20 & GDA & -5.34 \\
\hline PGA5 & -4.51 & PGA3 & -4.15 & PGA4 & -5.31 \\
\hline PGA3 & -4.50 & PGA4 & -4.08 & ADAMTS8 & -5.24 \\
\hline GJD2 & -4.49 & ADAMTS8 & -4.07 & TRAJ49 & -5.21 \\
\hline EDN2 & -4.20 & GJD2 & -4.07 & ADAMTS4 & -5.20 \\
\hline ETNPPL & -3.89 & NMUR1 & -3.88 & MT1A & -5.17 \\
\hline
\end{tabular}

$\mathrm{HF}$ — heart failure; DCM — dilated cardiomyopathy; DEGs — differentially expressed gene; RCM — restrictive cardiomyopathy

from +10.6 to $-5.5 \log 2 \mathrm{FC}$ between DCM and HD and from +6.4 to $-5.9 \log 2 \mathrm{FC}$ between $\mathrm{RCM}$ and HD. Most of DEGs (about 78\%, 61\%, and 65\%, respectively) were significantly overexpressed in $\mathrm{HF}, \mathrm{DCM}$, and RCM patients, indicating a strong induction in gene transcription. As shown in Table 2, the HAPLN1 gene is always up-regulated when $\mathrm{HF}$ was analyzed, DCM and RCM groups (log$2 \mathrm{FC}=+6.3 ;+6.2$ and +6.4 , respectively). Furthermore, the two most up-regulated genes were: CFAP61 and COL9A1 $(\log 2 \mathrm{FC}=+4.6$ and $+4.5)$ in HF group; MTRNR2L1 and MYOZ1 $(\log 2 \mathrm{FC}=+10.6$ and +5.3$)$ in DCM group; and CFAP61 and COL9A1 $(\log 2 \mathrm{FC}=+5.0$ for both genes) in RCM group. Similarly, the three most down-regulated genes were TRAJ39, TRAJ49, and CTRB1 $(\log 2 \mathrm{FC}=-5.3 ;-4.7$ and -4.7$)$ when HF vs. HD was analyzed; TRAJ39, CTRB1, and CXCL14 $(\log 2 \mathrm{FC}=-5.5 ;-4.9$ and -4.6$)$ in DCM vs. HD; and RSPO4, EDN2, and SELE $(\log 2 \mathrm{FC}=-5.9 ;-5.7$ and -5.7$)$ in $\mathrm{RCM}$ vs. control group (Table 2).
Interestingly, RNA-Seq analysis revealed the differential expression of 5 genes encoding different subunits of the mediator complex (MED): MED12, MED13L, MED14, MED17, MED23 (Fig. 1B; Table 3) (see the discussion section below).

Gene ontology and KEGG pathway enrichment analysis of DEGs

DAVID, Venny and Pathway analysis tools were used to determine molecular processes and biological pathways associated with the DEGs detected. We first associated differentially expressed mRNAs with three structured networks: biological process (BP), molecular function (MF) and cellular component (CC) in HF patients only (Tables S2-S5 - see journal website, supplementary file). Results showed a significant enrichment $(\mathrm{p}<0.05$ adjusted by the accepted Benjamini-Hochberg correction method) of particular biological processes when specific genes were altered. In particular, bioinformatic analysis revealed a significant alteration of "focal adhesion", 
Table 3. Differentially expressed genes of mediator subunits in heart failure (HF) versus heart donor (HD) comparison.

\begin{tabular}{|c|c|c|}
\hline Mediator subunit & HD & HF \\
\hline MED1 & 15.23 & 18.80 \\
\hline MED4 & 21.87 & 21.40 \\
\hline MED6 & 9.45 & 9.90 \\
\hline MED7 & 9.95 & 8.56 \\
\hline MED8 & 19.33 & 18.15 \\
\hline MED9 & 38.19 & 34.63 \\
\hline MED10 & 19.88 & 16.74 \\
\hline MED11 & 11.23 & 10.78 \\
\hline MED12 & 22.26 & 33.96 \\
\hline MED13 & 15.34 & 19.67 \\
\hline MED13L & 14.21 & 24.12 \\
\hline MED14 & 19.77 & 28.94 \\
\hline MED15 & 50.06 & 52.49 \\
\hline MED16 & 30.56 & 23.39 \\
\hline MED17 & 15.07 & 20.73 \\
\hline MED18 & 9.38 & 8.78 \\
\hline MED19 & 8.57 & 7.51 \\
\hline MED20 & 13.49 & 12.50 \\
\hline MED21 & 27.18 & 22.09 \\
\hline MED22 & 27.09 & 26.63 \\
\hline MED23 & 8.23 & 13.93 \\
\hline MED24 & 58.19 & 64.20 \\
\hline MED25 & 51.35 & 33.88 \\
\hline MED26 & 3.94 & 4.52 \\
\hline MED27 & 13.41 & 11.23 \\
\hline MED28 & 16.27 & 16.33 \\
\hline MED29 & 34.59 & 36.30 \\
\hline MED30 & 5.94 & 5.95 \\
\hline MED31 & 3.07 & 3.04 \\
\hline CDK8 & 8.29 & 11.83 \\
\hline CCNC & 15.90 & 18.48 \\
\hline
\end{tabular}

Gene expression values of mediator complex subunits expressed in counts per million. Differentially expressed gene of mediator complex subunits are shown in bold red color.

"regulation of actin cytoskeleton" and "oxidative phosphorylation" (adjusted p values 5.64 $4^{-11}, 2.30^{-1}$ and $1.27^{-12}$, respectively) (Fig. $1 \mathrm{C}$ ).

The GO analysis further validated this experimental set up, demonstrating that many biological processes and molecular functions, already associated with HF, were also enriched in these datasets, including "cell-adhesion", "cell-proliferation", "cell-differentiation”, "transcription”, “apoptosis”, "proteolysis”, etc. (Fig. 1D; Tables S2-S5). Next, by KEGG pathway analysis found a number of biological pathways characterized by up- or downregulated genes when under comparison (Fig. 2). The predominant up- and down-regulated pathways are summarized in Table S5, including related genes (345 up- and 239 down-regulated), and top 10 are represented in Figure 2A, B. Specific DEGs of the first two classes significantly altered are shown in Figure 2C-E (up-regulated DEGs) and Figure 2D-F (down-regulated DEGs).

Based on Pubmed literature, some genes which had not been previously reported showed an association with human heart tissue. Among them, for instance, ADAMTS8 and ADAMTSL4 were localized predominantly in plasma cells and lung tissue (Table S6 - see journal website, supplementary file) [36]. ADAMTS8 and ADAMTSL4 genes were particularly down-regulated in $\mathrm{HF}$ vs. HD $(-4.54$ fold; false discovery rate $[\mathrm{FDR}]<0.05$ and -2.62 fold; FDR $<0.05$ respectively). Both genes and many others in GO categories constitute an extremely rich source for potential target genes in $\mathrm{HF}$ understanding and prevention (Tables S2-S5). In addition, KEGG analysis demonstrated that several pathways were also altered in HF compared to HD; particularly in Figure 3A, were shown "cell adhesion molecules" pathway and graphically, heatmaps were also reported (Fig. 3B-C) of "focal adhesion" (39 up- and 9 down-regulated genes) and "cardiac muscle contraction" (3 up- and 20 down-regulated genes) significantly altered in all HF patients (Fig. 3A-C; Table S5). Finally, in order to identify a potential disease-specific signature, gene expression levels were compared in DCM and RCM groups. The Figure S1D-E (Fig. S1D-E - see journal website, supplementary file) shows the PCA of the distinct considered groups: DCM and $\mathrm{RCM}$ compared to HD, providing a quick display of overall variability in screening and can highlight inconsistent samples. 114 genes were found in which expression was significantly altered between the two conditions, using mean gene expression values of $\mathrm{HD}$ as the background condition (Fig. S2A-C see journal website, supplementary file). As shown in Figure S2D clusters of disease-specific genes belonging to the "TNF signaling pathway", "phagosome", "cytokine-cytokine receptor interaction" were identified.

In the analysis of DCM vs. HD, DEGs were 470 ranging between $\mathrm{FC}=-5.5$ (like TRAJ39 gene) and $\mathrm{FC}=+10.6$ (MTRNR2L1 gene). Of all DEGs about $61 \%$ (285 genes) were up-regulated and $39 \%$ (185 genes) were down-regulated. In analysis of RCM vs. HD, DEGs were 3,685 ranging between 


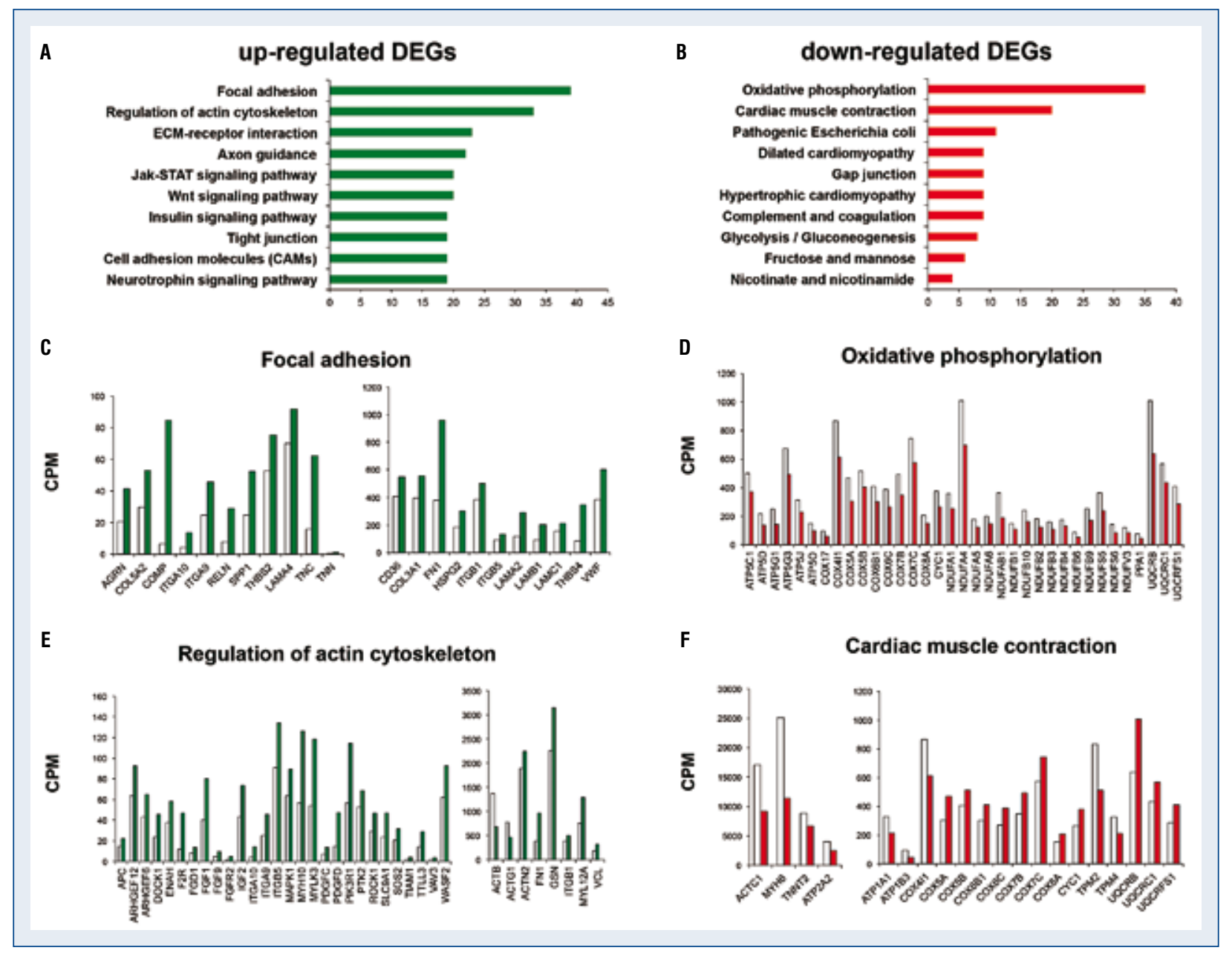

Figure 2. Pathway analysis of the top 10 significantly altered genes; A, B. Pathway analysis of the top 10 significantly changed up- and down-regulated differentially expressed gene (DEGs) when comparing heart failure and heart donor. In the panels $\mathbf{C} / \mathbf{E}$ and $\mathbf{D} / \mathbf{F}$ are reported specific up- and down-regulated DEGs of the first and second more abundant pathway; CPM - counts per million.

a $\mathrm{FC}=-5.9$ (like $\mathrm{RSPO} 4$ gene) and $\mathrm{FC}=+6.4$ (HAPLN1 gene). Of all DEGs about 65\% (2,390 genes) were up-regulated and 35\% (1,295 genes) were down-regulated.

The similarly of HF vs. HD comparisons, DCM and RCM DEGs compared to non-failing hearts were examined by GO and KEGG enrichment analyses separately. DEGs with higher expression levels in DCM were enriched for "cardiovascular" $\left(\mathrm{p}=5.0 \times 10^{-5}\right)$ in disease class and were enriched for "cardiac muscle contraction" $\left(\mathrm{p}=2.0 \times 10^{-6}\right)$ in KEGG pathway. The GO analysis showed that the DEGs clustered in $\mathrm{MF}, \mathrm{BP}$, and $\mathrm{CC}$ and were significantly enriched for terms related to "extracellular region" $\left(\mathrm{p}=1.6 \times 10^{-13}\right)$ and "cell junction" $\left(\mathrm{p}=3.3 \times 10^{-4}\right)$. Similarly, DEGs with higher expression levels in RCM were enriched for "cardio- vascular" $\left(\mathrm{p}=2.4 \times 10^{-12}\right)$ in disease class. Instead, KEGG analysis was significantly enriched for "focal adhesion" $\left(\mathrm{p}=1.6 \times 10^{-3}\right)$ and "ECM-receptor interaction" ( $\left.p=2.9 \times 10^{-3}\right)$. Finally, GO analysis showed that RCM DEGs clustered in MF, BP, and $\mathrm{CC}$ and were significantly enriched for terms related to "cell adhesion molecules" $\left(\mathrm{p}=3.9 \times 10^{-3}\right)$ and "cardiac muscle contraction" $\left(\mathrm{p}=1.3 \times 10^{-2}\right)$.

Transcriptome analysis of failing hearts identifies differentially expressed lncRNAs

Since lncRNA are emerging as crucial contributors to human diseases, the aim of this investigation was whether these non-coding RNAs may potentially have a role in HF. Therefore a systematic analysis of RNA-Seq datasets to identify lncRNAs differentially expressed (DE) was undertaken in 


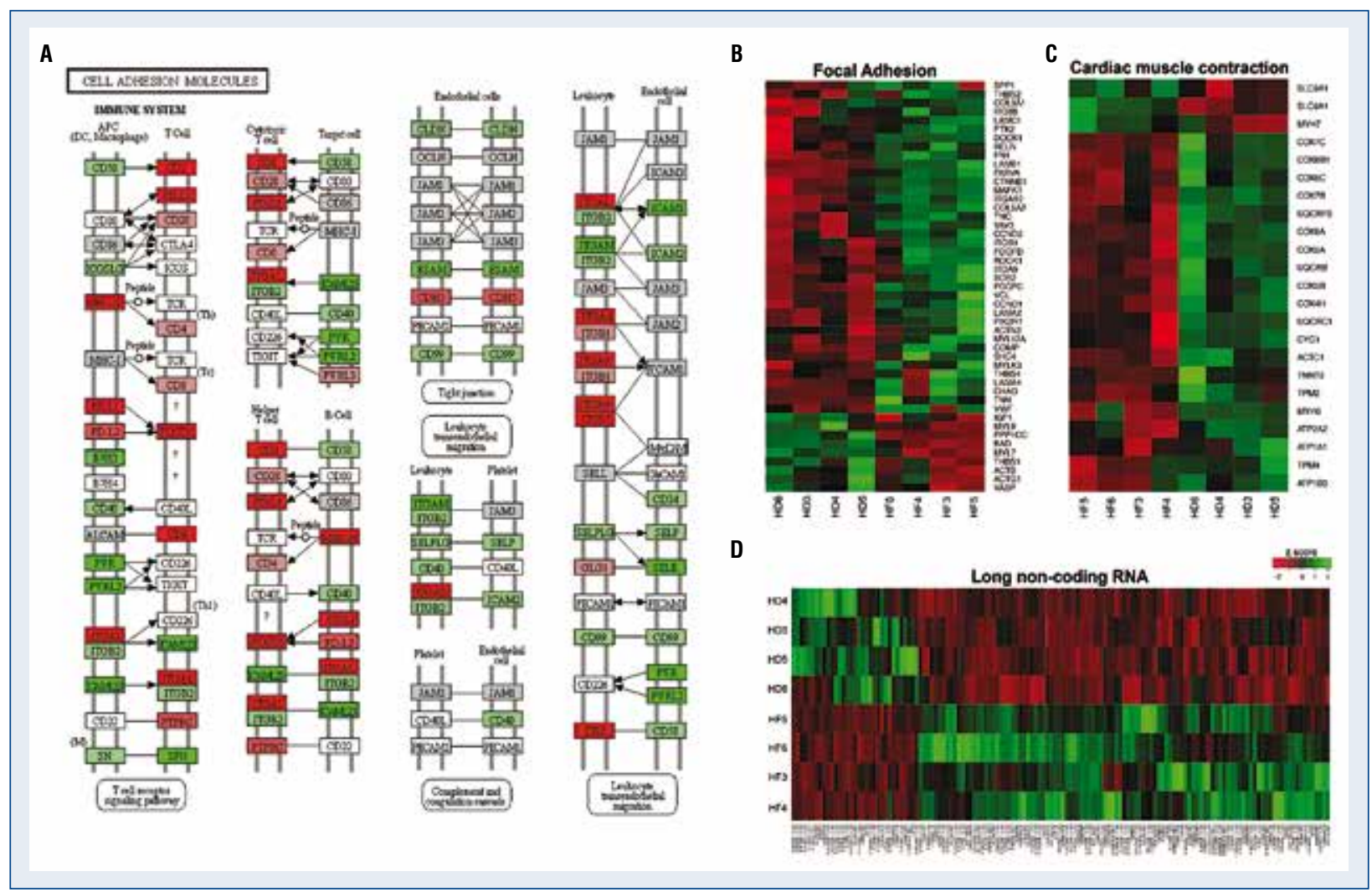

Figure 3. Pathways most affected in heart failure (HF) versus heart donor (HD) from RNA sequencing analysis. A. Graphical representation of the KEGG pathway "Cell adhesion molecules". Red boxes indicate genes down-regulated in HF, green the ones up-regulated. "Focal adhesion" and "Cardiac muscle contraction" pathway are reported in the panels $\mathbf{B}$ and $\mathbf{C}$ as heatmaps (with the hierarchical clustering option). The degree of differential expression between the two HF and HD groups is indicated by a three-color code (down-regulated genes are depicted in red, up-regulated genes in green and genes with little-to-none variation are indicated in black). Similarly, panel D shows the heatmap (with the hierarchical clustering option) of long non-coding RNAs differentially expressed in HF versus HD samples.

HF vs. HD. In this data-driven analysis. Focus was mainly on intergenic and antisense lncRNAs, which do not overlap protein-coding loci and that can be unambiguously quantified using RNA-Seq. Differential expression analysis revealed that 140 lncRNA were significantly deregulated in HF vs. HD. Among them, 33 non-coding genes were upregulated and 107 were down-regulated (Fig. 3D; Table 4). As lncRNA may act to regulate gene expression of surrounding protein-coding genes, DE $\operatorname{lncRNAs}$ were associated, through an automatic computational pipeline, with the transcription start site of the nearest protein-coding gene. Of these, 27 (24 up- and 3 down-regulated) lncRNAs were DE (Table 4). Interestingly, among the most altered lncRNAs/gene and lincRNA/gene pairs we found EPHA5-AS1/EPHA5 and JAK1/ /RP11-182I10 genes, respectively. EPHA5-AS1 is located on chromosome 4q13.2 and Ensembl annotates 3 different isoforms transcribed from this locus, with a long isoform composed of 4 exons ( 1,177 nucleotide in length). Moreover, EPHA5-AS1 has a partial overlap with the 5'UTR of EPHA5 gene (Fig. 4A), suggesting a direct regulatory role of this antisense lncRNA on the sense protein-coding gene. To address whether a potential correlation may exist, normalized expression values of both the protein-coding and the lncRNA in all samples were compared. Interestingly, it was observed that both of them were significantly up-regulated in HF compared to HD (Fig. 4B), and the expression values had a high positive correlation (Pearson's coefficient $=0.89$ ). Also similarly revealed, was a positive correlation between JAK1 and RP11-182I10, a lincRNA/mRNA transcribed in the same orientation of JAK1 gene and was localized very close to this gene (Fig. 4C). Boxplots in Figure 4D show the expression values of both genes under two different conditions (FDR $<0.05$ ). 
Table 4. Long noncoding RNAs (IncRNA) differentially expressed (DE) in heart failure versus heart donor groups.

\begin{tabular}{lccc}
\hline DE IncRNA & DE associated-protein-coding gene & IncRNA log2FC & Protein-coding log2FC \\
\hline RP11-427H3 & AAK1 & 1.25 & 1.34 \\
LINC00342 & ANKRD36C & 0.87 & 0.85 \\
LUCAT1 & ARRDC3 & -1.34 & 0.81 \\
LINC00900 & CADM1 & 1.28 & 1.06 \\
SFTA1P & CELF2 & 2.57 & 0.50 \\
RP11-261C10 & CEP170 & 0.95 & 0.72 \\
RP11-807H7 & EPHA5 & 1.20 & 1.27 \\
RP11-479J7 & FAM78B & -3.28 & -3.07 \\
GDNF-AS1 & GDNF & 2.58 & 1.84 \\
RP11-441015 & GOT1 & 1.30 & -0.32 \\
AC018647 & HERPUD2 & 2.10 & 0.52 \\
RP11-182I10 & JAK1 & 1.10 & 0.39 \\
AC096574 & LRRFIP1 & 1.81 & 0.59 \\
CR0CCP2 & NBPF1 & 0.60 & 0.55 \\
RP11-121C2 & NFXL1 & 1.14 & -2.70 \\
LINC01011 & NQO2 & 0.72 & -0.51 \\
AC010096 & OSR1 & 0.65 & -1.43 \\
RP11-33B1 & PDE5A & 1.06 & 0.65 \\
RP11-10L7 & PPM1K & 1.39 & 1.42 \\
RP11-1114A5 & RBMX & 0.75 & 0.38 \\
NEAT1 & SLC25A45 & -0.47 & 1.01 \\
RP11-480A16 & TNK2 & 0.82 & 0.77 \\
RP11-1275H24 & TNRC18 & 0.62 & 0.61 \\
USP46-AS1 & USP46 & 0.89 & 0.95 \\
OVAAL & XPR1 & 1.60 & 1.31 \\
MIRLET7DHG & ZNF169 & 0.97 & 0.93 \\
RP11-457M11 & ZNF322 & 0.97 & \\
\hline
\end{tabular}

\section{RNA-Seq validation}

To validate the results of the analysis between two conditions, qRT-PCR on the same RNAs used for RNA-Seq experiments were performed (Table S1). Under analysis was the expression of several selections of significantly altered genes, including all GO categories, comparing HF and HD groups (Tables S2-S5). Particularly, altered cytoskeletal components, closely involved in the regulation of the actin and myosin cytoskeleton and muscle contraction, included: ACTA2, ACTG2, NMUR1, MYL4, and MYH10. Moreover, the following transcription factors involved in heart development and morphogenesis were selected: TBX20, SHOX2, HOPX and MSX1. Furthermore, among significantly DEGs, the retinoic acid producing enzyme ALDH1A2 was chosen for validation. Finally, among genes encoding for ionic channels, we selected: SLC8A1, CHRNE, HCN2, BDKRB2, and CACNA1G.

All DEGs analyzed by qRT-PCR confirmed the expression trends obtained by RNA-Seq analysis. In Figure S3 (see journal website, supplementary file) observation revealed that FC measured by RNA-Seq (count per million [CPM]) was confirmed also by qRT-PCR $\left(2^{-\Delta \Delta \mathrm{Ct}}\right)$.

\section{Discussion}

Although a limited number of patients participated, the main findings of the present study were:

1. The identification of specific changes occurring in the transcriptome of $\mathrm{HF}$ patients 


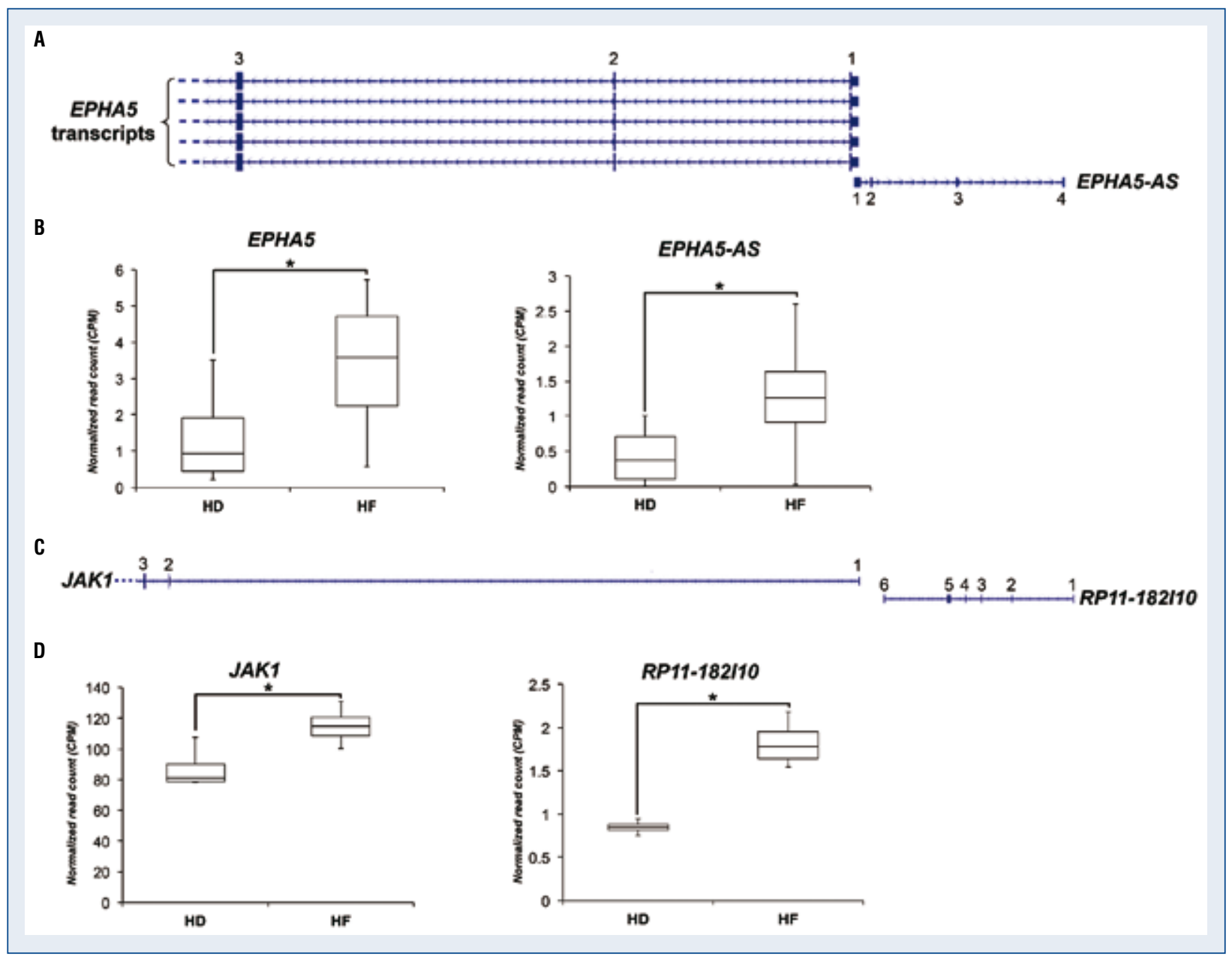

Figure 4. Genomic localization of differentially expressed antisense long noncoding RNA (IncRNAs); panels A (EPHA5/ /EPHA5-AS1) and C (JAK1/RP11-182I10) show the schematic graphical representations of IncRNA/mRNA and long intergenic RNAs (lincRNAs)/mRNA pairs (RefSeq genes) that are significantly altered in heart failure patients. In panels $\mathbf{B}$ and $\mathbf{D}$ the expression levels (normalized values, counts per million [CPM]) of both the gene and the neighbor IncRNA associated to it (Pearson's coefficient $=0.89$ ) are reported (FDR $<0.05)$; HD — heart donor; HF — heart failure.

compared to healthy subjects. In particular, bioinformatic analysis revealed a significant alteration of "focal adhesion", "regulation of actin cytoskeleton" and "oxidative phosphorylation" in HF cardiomyocytes;

2. The identification of some genes, which had not been previously reported, had an association with human heart tissue and $\mathrm{HF}$ (ADAMTS8, ADAMTSL4, ACTA2, ACTG2 and NMUR1);

3. The identification of a potential disease-specific signature, comparing gene expression levels in DCM and RCM groups, revealing as significantly DEGs, genes belonging to the "TNF signaling pathway", "phagosome", "cytokine-cytokine receptor interaction";
4. The identification of altered levels of genes encoding 5 MED subunits that play a fundamental role in human HF. Among these, to date, MED17 has not yet been associated with CVD;

5. The identification of specific non-coding RNAs that potentially have a role in HF. Focusing mainly on intergenic and antisense lncRNAs, differential expression analysis revealed that EPHA5-AS1/EPHA5 and JAK1/RP11-182I10 genes (a lncRNAs/gene and lincRNA/gene pairs respectively) were significantly deregulated in HF vs. HD.

Cardiomyocyte cytoskeleton is essential to maintain cell morphology and regulate contraction and relaxation. Alterations in cytoskeletal 
components have been found in both animal models of $\mathrm{HF}$ and in humans [37, 38]. Specifically, an increase in cytoskeletal proteins together with a loss of contractile filaments has been suggested as the morphological cause of heart dysfunction $[37,38]$. The number of genes involved in this structural disruption continues increasing; however, other cytoskeleton and contractile fiber genes are expected to play a role in these pathologies. As previously reported, most genes encoding components for a wide variety of cellular compartments and pathways, such as: contractile and transduction apparatus, gene transcription and splicing machinery, and calcium regulation, were associated with DCM [11]. An example of altered genes, also deregulated in this condition and was represented by ACTC1, DSG2, MYH6, SGCD (Tables S2-S5). On the other hand, genetic mutations in familial RCM are not well defined, since there is significant overlap in the mutations between RCM, DCM, and HCM [12]. Also in RCM, accordingly to previous studies and presently identified genes encoding sarcomere (e.g. ACTC1, MYH7, and TNNT2), Z-disk proteins (e.g. MYPN), and intermediate filament network (e.g. DES) (Tables S2-S5). Interestingly, in the present study, a large number of genes were identified that had not been previously correlated with HF. Although the specific GO category/term is strongly associated with this pathology, significantly altered genes were found associated to "myocardial infarction susceptibility" and "cardiomyopathy" only when down-modulated genes were considered. DEGs were also significantly enriched in "immune response" and "inflammation-related" pathways. Furthermore, other genes involved in "angiogenesis" and "cytoskeleton" were significantly altered in $\mathrm{HF}$ vs. HD as well as some genes with very important biochemical/biological functions in other tissues or organs (Table S6). The list of genes includes: ACTA2, NMUR1, ACTG2, KCNJ5, GATA6, MSX1, ZNF385B, ADAMTS8, ADAMTSL4 and COMP. In particular, ACTA2 and ACTG2 have been associated with $\mathrm{HF}$ for the first time in this study. Specifically, these genes were down-regulated both in DCM and $\mathrm{RCM}$ conditions. In the analysis of DCM and RCM vs. control group, ACTA2 gene reported a $\mathrm{FC}=-2.7$ and -3.3 , respectively. This trend was also confirmed for ACTG2, with a FC $=-8.2$ and -7.5 in DCM and RCM, respectively. As previously reported, these genes encode highly conserved molecules belonging to the actin family proteins, involved in cell motility, structure and integrity of the contractile apparatus [39]. Noteworthy, NMUR1 gene is particularly down-regulated in HF $(-3.78$ fold; FDR $<0.05)$; this gene is endogenously expressed in adult rat cardiomyocytes, where it is involved in modulating L-type $\mathrm{Ca}(2+)$ channels [40]. Thus, this study suggests that the altered levels of NMUR1 may also play a significant role in human HF.

Generally, the findings of this study indicate a more complex transcriptome alteration in RCM compared to DCM. Indeed, in DCM filtered list a total of 50 genes which were differentially expressed whereas RCM tissues displayed 516 common DEGs. Nevertheless, despite the different etiologies of DCM and RCM, it is conceivable that both pathologies can share similar functional changes that are responsible for HF.

Moreover, bioinformatic functional analysis demonstrated that DEGs binding-related genes (1,892 up-regulated and 536 down-regulated genes) were differentially expressed in failing samples when compared with non-failing hearts (Fig. 2A).

Interestingly, our analysis also revealed the differential expression of 5 genes encoding various subunits of the MED: MED12, MED13L, MED14, MED17, MED23 when a PP $>0.95$ was used to determine DEGs (Fig. 1B; Table 3). To date, alterations in MED complex genes have been associated with several human multifactorial diseases, including CVDs [41-44]. To date, only MED17 has not yet been associated with CVD. All differentially expressed MED were up-regulated in HF patients. In particular, MED12 expression was found altered in DCM and RCM groups when we used a PP $>0.80$. Noteworthy, MED17 gene is up-regulated in RCM patients $(+0.50$ fold; FDR $<0.05)$; this gene constitute a subunit of MED head module and interact with Pol II and the general transcription factors for both transcription and nucleotide excision repair mechanisms [45]. Thus, our pilot study suggests that the altered levels of MED subunits may play a fundamental role in human HF. In particular, since that MED complex has been also shown to work in cooperation with ncRNAs in regulating gene transcription [41], it would be relevant to investigate MED17 functions in order to understand its role in the onset and progression of RCM.

To date, several reports indicate that other classes of RNAs, other than protein-coding, are potential contributors to human diseases [46, 47], mainly as they are able to regulate gene expression at the transcriptional, post-transcriptional, and epigenetic levels [48, 49]. Among these ncRNAs, the class of lncRNAs plays an important role in several conditions such as cancer, liver disease and central nervous system disorders among many others. Exploratory studies performed on the lncRNA in 
the cardiovascular setting have thus far identified only few lncRNA associated with CVDs [50, 51]. Specific studies looking at lncRNAs in HF remain lacking [51].

To investigate whether lncRNAs may potentially have a role in heart diseases, systematic analysis of RNA-Seq datasets to identify lncRNAs $\mathrm{DE}$ in HF was performed. Differential expression analysis revealed 140 significantly deregulated lncRNA in HF vs. HD. Among them, $24 \%$ of noncoding genes were up-regulated and $76 \%$ were down-regulated. A searched for all the $140 \mathrm{DE}$ lncRNAs the nearest protein-coding genes and we could find only 27 pairs of lncRNA-genes that are both differentially expressed was done. Of all, 27 genes (about 20\%) were DE (Table 4). Interestingly, among associated DE 1 ncRNAs and protein coding genes, we found EPHA5-AS1 (annotated also as RP11-807H7.1), a lncRNA transcribed antisense to EPHA5 gene. Both the protein-coding and the lncRNA are up-regulated in HF vs. HD (Fig. 4). Moreover, EPHA5-AS1 has a partial overlap with the 5'UTR of EPHA5 gene (Fig. 4), which suggests a direct regulatory role of this antisense lncRNA on the sense protein-coding gene. Ephrin receptors have diverse activities, including widespread effects on the actin cytoskeleton, cell-substrate adhesion, intercellular junctions, cell shape, and cell movement [52]. However, this is the first report about its deregulation in cardiomyopathies.

Similarly, a positive correlation between JAK1 and the lincRNA RP11-182I10 was observed. Different from EPHA5-AS1, RP11-182I10 is transcribed on the same strand of JAK1 gene in a typical headto-tail orientation (Fig. 4C). The expression values of both genes under two different conditions were also increased (FDR < 0.05) (Fig. 4D). JAK1 gene encodes a protein-tyrosine kinase member, operating fundamental roles as the intracellular signaling effector of cytokine receptors. The activation and/or inactivation of members of the Janus kinase family are causally linked to different human diseases, as hemopoietic malignancies, immunodeficiency and inflammatory diseases [53]. However, EPHA5 and the JAK1 gene have never been identified as altered in patients with cardiomyopathies. These novel changes could be responsible for altered contraction and cell disruption in HF subjects, which may suggest novel therapeutic approaches.

\section{Conclusions}

The present findings revealed specific expression pattern of both protein-coding and lncRNAs in HF patients, confirming that new LV myocardial biomarkers could be reliably identified using NextGeneration Sequencing-based approaches.

For patients awaiting heart transplantation, there are differences in survival on the basis of type of heart disease (DCM or RCM). The knowledge of an expression network signature in end-stage HF diseased hearts may offer important insights into the complex pathogenesis of advanced cardiac failure, it may also provide potential targets for therapeutic intervention.

All the novel changes revealed could be responsible for altered contraction and cell disruption in HF subjects. However, many of these factors, play critical roles in heart development and homeostasis as well as in other human diseases, an original list of novel candidate genes with potential implications in HF is offered. These findings reveal a specific expression pattern of both protein-coding and lncRNAs in HF patients, confirming that new LV myocardial biomarkers can be reliably identified using NGS-based approaches. Interestingly, for many of these proteins; pharmacological agonists and antagonists could be developed, raising exciting possibilities for new therapeutic approaches.

\section{Acknowledgements}

We thank Dr. Casamassimi and Dr. Rienzo (Department of Biochemistry, Biophysics and General Pathology, Second University of Naples, Italy) for their valuable technical support in the first steps of this study.

This work was supported by grants from "Ministero della Salute" [project code GR-201102349436] and "RRC 2013-2015" [project code RRC-2015-2360454].

\section{Conflict of interest: None declared}

\section{References}

1. Modesto K, Sengupta PP. Myocardial mechanics in cardiomyopathies. Prog Cardiovasc Dis. 2014; 57(1): 111-124, doi: 10.1016/j. pcad.2014.03.003, indexed in Pubmed: 25081406.

2. McMurray J, Adamopoulos S, Anker SD, et al. ESC Guidelines for the diagnosis and treatment of acute and chronic heart failure 2012: The Task Force for the Diagnosis and Treatment of Acute and Chronic Heart Failure 2012 of the European Society of Cardiology. Developed in collaboration with the Heart Failure Association (HFA) of the ESC. European Heart Journal. 2012; 33(14): 1787-1847, doi: 10.1093/eurheartj/ehs104.

3. Roger VL. Epidemiology of heart failure. Circ Res. 2013; 113(6): 646-659, doi: 10.1161/CIRCRESAHA.113.300268, indexed in Pubmed: 23989710.

4. Ziaeian B, Fonarow G. Epidemiology and aetiology of heart failure. Nat Rev Cardiol. 2016; 13(6): 368-378, doi: 10.1038/nrcardio.2016.25. 
5. Matkovich SJ, Zhang Y, Van Booven DJ, et al. Deep mRNA sequencing for in vivo functional analysis of cardiac transcriptional regulators: application to Galphaq. Circ Res. 2010; 106(9): 1459-1467, doi: 10.1161/CIRCRESAHA.110.217513, indexed in Pubmed: 20360248

6. Napoli C, Lerman LO, Sica V, et al. Microarray analysis: a novel research tool for cardiovascular scientists and physicians. Heart. 2003; 89(6): 597-604, doi: 10.1136/heart.89.6.597, indexed in Pubmed: 12748210.

7. Gora M, Kiliszek M, Burzynska B. Will global transcriptome analysis allow the detection of novel prognostic markers in coronary artery disease and heart failure? Curr Genomics. 2013; 14(6): 388-396, doi: 10.2174/1389202911314090006, indexed in Pubmed: 24396272.

8. Nagalakshmi U, Wang Z, Waern K, et al. The transcriptional landscape of the yeast genome defined by RNA sequencing. Science. 2008; 320(5881): 1344-1349, doi: 10.1126/science.1158441, indexed in Pubmed: 18451266.

9. Costa V, Aprile M, Esposito R, et al. RNA-Seq and human complex diseases: recent accomplishments and future perspectives. Eur J Hum Genet. 2013; 21(2): 134-142, doi: 10.1038/ ejhg.2012.129, indexed in Pubmed: 22739340.

10. Herrer I, Roselló-Lletí E, Rivera M, et al. RNA-sequencing analysis reveals new alterations in cardiomyocyte cytoskeletal genes in patients with heart failure. Lab Invest. 2014; 94(6): 645-653, doi: 10.1038/labinvest.2014.54, indexed in Pubmed: 24709777.

11. Hershberger RE, Siegfried JD. Update 2011: clinical and genetic issues in familial dilated cardiomyopathy. J Am Coll Cardiol. 2011; 57(16): 1641-1649, doi: 10.1016/j.jacc.2011.01.015, indexed in Pubmed: 21492761.

12. Towbin J. Inherited Cardiomyopathies. Circulation Journal. 2014; 78(10): 2347-2356, doi: 10.1253/circj.cj-14-0893.

13. Ounzain S, Micheletti R, Beckmann T, et al. Genome-wide profiling of the cardiac transcriptome after myocardial infarction identifies novel heart-specific long non-coding RNAs. Eur Heart J. 2015; 36(6): 353-68a, doi: 10.1093/eurheartj/ehu180, indexed in Pubmed: 24786300.

14. di Salvo TG, Yang KC, Brittain E, et al. Right ventricular myocardial biomarkers in human heart failure. J Card Fail. 2015; 21(5): 398-411, doi: 10.1016/j.cardfail.2015.02.005, indexed in Pubmed: 25725476.

15. He M, Yang $Z$, Abdellatif M, et al. GTPase Activating Protein (Sh3 Domain) Binding Protein 1 Regulates the Processing of MicroRNA-1 during Cardiac Hypertrophy. PLoS One. 2015; 10(12): e0145112, doi: 10.1371/journal.pone.0145112, indexed in Pubmed: 26675618.

16. Chen S, Puthanveetil P, Feng B, et al. Cardiac miR-133a overexpression prevents early cardiac fibrosis in diabetes. J Cell Mol Med. 2014; 18(3): 415-421, doi: 10.1111/jcmm.12218, indexed in Pubmed: 24428157.

17. Ganesan J, Ramanujam D, Sassi Y, et al. MiR-378 controls cardiac hypertrophy by combined repression of mitogen-activated protein kinase pathway factors. Circulation. 2013; 127(21): 20972106, doi: 10.1161/CIRCULATIONAHA.112.000882, indexed in Pubmed: 23625957.

18. Heymans S, Corsten MF, Verhesen W, et al. Macrophage microRNA-155 promotes cardiac hypertrophy and failure. Circulation. 2013; 128(13): 1420-1432, doi: 10.1161/CIRCULATIONAHA.112.001357, indexed in Pubmed: 23956210.

19. Ucar A, Gupta SK, Fiedler J, et al. The miRNA-212/132 family regulates both cardiac hypertrophy and cardiomyocyte autoph- agy. Nat Commun. 2012; 3: 1078, doi: 10.1038/ncomms2090, indexed in Pubmed: 23011132.

20. da Costa Martins PA, Salic K, Gladka MM, et al. MicroRNA199b targets the nuclear kinase Dyrk1a in an auto-amplification loop promoting calcineurin/NFAT signalling. Nat Cell Biol. 2010; 12(12): 1220-1227, doi: 10.1038/ncb2126, indexed in Pubmed: 21102440.

21. Han $\mathrm{P}, \mathrm{Li} \mathrm{W}$, Lin $\mathrm{CH}$, et al. A long noncoding RNA protects the heart from pathological hypertrophy. Nature. 2014; 514(7520): 102-106, doi: 10.1038/nature13596, indexed in Pubmed: 25119045.

22. Kumarswamy R, Bauters C, Volkmann I, et al. Circulating long noncoding RNA, LIPCAR, predicts survival in patients with heart failure. Circ Res. 2014; 114(10): 1569-1575, doi: 10.1161/ CIRCRESAHA.114.303915, indexed in Pubmed: 24663402.

23. Vausort M, Wagner DR, Devaux Y. Long noncoding RNAs in patients with acute myocardial infarction. Circ Res. 2014; 115(7): 668-677, doi: 10.1161/CIRCRESAHA.115.303836, indexed in Pubmed: 25035150.

24. Molina-Navarro MM, Roselló-Lletí E, Tarazón E, et al. Heart failure entails significant changes in human nucleocytoplasmic transport gene expression. Int J Cardiol. 2013; 168(3): 2837-2843, doi: 10.1016/j.ijcard.2013.03.192, indexed in Pubmed: 23651824 .

25. Rienzo M, Costa V, Scarpato M, et al. RNA-Seq for the identification of novel Mediator transcripts in endothelial progenitor cells. Gene. 2014; 547(1): 98-105, doi: 10.1016/j.gene.2014.06.034, indexed in Pubmed: 24952135.

26. Kim D, Pertea G, Trapnell C, et al. TopHat2: accurate alignment of transcriptomes in the presence of insertions, deletions and gene fusions. Genome Biol. 2013; 14(4): R36, doi: 10.1186/gb2013-14-4-r36, indexed in Pubmed: 23618408.

27. Russo F, Angelini C. RNASeqGUI: a GUI for analysing RNA-Seq data. Bioinformatics. 2014; 30(17): 2514-2516, doi: 10.1093/bioinformatics/btu308, indexed in Pubmed: 24812338.

28. Soneson C, Delorenzi M. A comparison of methods for differential expression analysis of RNA-seq data. BMC Bioinformatics. 2013; 14: 91, doi: 10.1186/1471-2105-14-91, indexed in Pubmed: 23497356.

29. Kanehisa M. KEGG: Kyoto Encyclopedia of Genes and Genomes. Nucleic Acids Res. 2000; 28(1): 27-30, doi: 10.1093/nar/28.1.27.

30. Huang DaW, Sherman BT, Tan Q, et al. The DAVID Gene Functional Classification Tool: a novel biological module-centric algorithm to functionally analyze large gene lists. Genome Biol. 2007; 8(9): R183, doi: 10.1186/gb-2007-8-9-r183, indexed in Pubmed: 17784955.

31. Mi H, Muruganujan A, Thomas PD. PANTHER in 2013: modeling the evolution of gene function, and other gene attributes, in the context of phylogenetic trees. Nucleic Acids Res. 2013; 41(Database issue): D377-D386, doi: 10.1093/nar/gks1118, indexed in Pubmed: 23193289.

32. Karolchik D, Hinrichs AS, Furey TS, et al. The UCSC Table Browser data retrieval tool. Nucleic Acids Res. 2004; 32 (Database issue): D493-D496, doi: 10.1093/nar/gkh103, indexed in Pubmed: 14681465.

33. Quinlan AR, Hall IM. BEDTools: a flexible suite of utilities for comparing genomic features. Bioinformatics. 2010; 26(6): 841-842, doi: 10.1093/bioinformatics/btq033, indexed in Pubmed: 20110278.

34. Rienzo M, Schiano C, Casamassimi A, et al. Identification of valid reference housekeeping genes for gene expression analysis 
in tumor neovascularization studies. Clin Transl Oncol. 2013; 15(3): 211-218, doi: 10.1007/s12094-012-0904-1, indexed in Pubmed: 22855186 .

35. Costa V, Angelini C, De Feis I, et al. Uncovering the complexity of transcriptomes with RNA-Seq. J Biomed Biotechnol. 2010; 2010: 853916, doi: 10.1155/2010/853916, indexed in Pubmed: 20625424.

36. Kelwick R, Desanlis I, Wheeler GN, et al. The ADAMTS (A Disintegrin and Metalloproteinase with Thrombospondin motifs) family. Genome Biol. 2015; 16: 113, doi: 10.1186/s13059-0150676-3, indexed in Pubmed: 26025392.

37. Kostin S, Hein S, Arnon E, et al. The cytoskeleton and related proteins in the human failing heart. Heart Fail Rev. 2000; 5(3): 271-280, doi: 10.1023/A:1009813621103, indexed in Pubmed: 16228910 .

38. Zile MR, Green GR, Schuyler GT, et al. Cardiocyte cytoskeleton in patients with left ventricular pressure overload hypertrophy. J Am Coll Cardiol. 2001; 37(4): 1080-1084, indexed in Pubmed: 11263612 .

39. Guo DC, Pannu H, Tran-Fadulu V, et al. Mutations in smooth muscle alpha-actin (ACTA2) lead to thoracic aortic aneurysms and dissections. Nat Genet. 2007; 39(12): 1488-1493, doi: 10.1038/ng.2007.6, indexed in Pubmed: 17994018.

40. Chen RX, Liu F, Li Y, et al. Neuromedin S increases L-type $\mathrm{Ca}(2+)$ channel currents through $\mathrm{G}(\mathrm{i}) \alpha$-protein and phospholipase $\mathrm{C}$-dependent novel protein kinase $\mathrm{C}$ delta pathway in adult rat ventricular myocytes. Cell Physiol Biochem. 2012; 30(3): 618-630, doi: 10.1159/000341443, indexed in Pubmed: 22832358.

41. Schiano C, Casamassimi A, Vietri MT, et al. The roles of mediator complex in cardiovascular diseases. Biochim Biophys Acta. 2014; 1839(6): 444-451, doi: 10.1016/j.bbagrm.2014.04.012, indexed in Pubmed: 24751643.

42. Muncke N, Jung C, Rüdiger H, et al. Missense mutations and gene interruption in PROSIT240, a novel TRAP240-like gene, in patients with congenital heart defect (transposition of the great arteries). Circulation. 2003; 108(23): 2843-2850, doi: 10.1161/01. CIR.0000103684.77636.CD, indexed in Pubmed: 14638541.

43. Grøntved L, Madsen MS, Boergesen M, et al. MED14 tethers mediator to the $\mathrm{N}$-terminal domain of peroxisome proliferator- activated receptor gamma and is required for full transcriptional activity and adipogenesis. Mol Cell Biol. 2010; 30(9): 2155-2169, doi: 10.1128/MCB.01238-09, indexed in Pubmed: 20194623.

44. Wang W, Huang Lu, Huang Y, et al. Mediator MED23 links insulin signaling to the adipogenesis transcription cascade. Dev Cell. 2009; 16(5): 764-771, doi: 10.1016/j.devcel.2009.04.006, indexed in Pubmed: 19460352.

45. Kikuchi Y, Umemura H, Nishitani S, et al. Human mediator MED17 subunit plays essential roles in gene regulation by associating with the transcription and DNA repair machineries. Genes Cells. 2015; 20(3): 191-202, doi: 10.1111/gtc.12210, indexed in Pubmed: 25482373.

46. Femminella GD, Ferrara N, Rengo G. The emerging role of microRNAs in Alzheimer's disease. Front Physiol. 2015; 6: 40, doi: 10.3389/fphys.2015.00040, indexed in Pubmed: 25729367.

47. Clarke BD, Roby JA, Slonchak A, et al. Functional non-coding RNAs derived from the flavivirus 3' untranslated region. Virus Res. 2015; 206: 53-61, doi: 10.1016/j.virusres.2015.01.026, indexed in Pubmed: 25660582.

48. Iyer MK, Niknafs YS, Malik R, et al. The landscape of long noncoding RNAs in the human transcriptome. Nat Genet. 2015; 47(3): 199-208, doi: 10.1038/ng.3192, indexed in Pubmed: 25599403

49. Juan L, Wang G, Radovich M, et al. Potential roles of microRNAs in regulating long intergenic noncoding RNAs. BMC Med Genomics. 2013; 6 Suppl 1: S7, doi: 10.1186/1755-8794-6-S1-S7, indexed in Pubmed: 23369519.

50. Skroblin P, Mayr M. Circ Res. 2014; 115(7): 607-609, doi: 10.1161/circresaha.114.304839.

51. Philippen LE, Dirkx E, da Costa-Martins PA, et al. Non-coding RNA in control of gene regulatory programs in cardiac development and disease. J Mol Cell Cardiol. 2015; 89(Pt A): 51-58, doi: 10.1016/j.yjmcc.2015.03.014, indexed in Pubmed: 25820097.

52. Pasquale EB. Eph-ephrin bidirectional signaling in physiology and disease. Cell. 2008; 133(1): 38-52, doi: 10.1016/j. cell.2008.03.011, indexed in Pubmed: 18394988.

53. Babon JJ, Lucet IS, Murphy JM, et al. The molecular regulation of Janus kinase (JAK) activation. Biochem J. 2014; 462(1): 1-13, doi: 10.1042/BJ20140712, indexed in Pubmed: 25057888. 